\title{
A COMPLEX ANALOGUE OF THE ROLLE THEOREM AND POLYNOMIAL ENVELOPES OF IRREDUCIBLE DIFFERENTIAL EQUATIONS IN THE COMPLEX DOMAIN
}

\author{
D. NOVIKOV AND S. YAKOVENKO
}

\begin{abstract}
We prove a complex analytic analogue of the classical Rolle theorem asserting that the number of zeros of a real smooth function can exceed that of its derivative by at most 1 . This result is used then to obtain upper bounds for the number of complex isolated zeros of:

(1) functions defined by linear ordinary differential equations (in terms of the magnitude of the coefficients of the equations);

(2) elements from the polynomial envelope of a linear differential equation with an irreducible monodromy group (in terms of the degree of the envelope);

(3) successive derivatives of a function defined by a linear irreducible equation (in terms of the order of the derivative).

These results generalize the bounds from $[2,5,6]$ that were previously obtained for the number of real isolated zeros.
\end{abstract}

\section{Introduction}

1.1. Zeros of functions defined by linear ordinary differential equations with holomorphic coefficients

Let $L$ be an ordinary linear differential operator with rational coefficients,

$$
L=\partial^{n}+a_{1}(t) \partial^{n-1}+\cdots+a_{n-1}(t) \partial+a_{n}(t), \quad \text { where } t \in \mathbb{C}, \partial=\frac{d}{d t}, a_{k} \in \mathbb{C}(t),
$$

and $\Sigma_{L} \subset \mathbb{C} P^{1}$ is its singular locus (the polar set for the coefficients with the point $t=\infty$ added if, in the chart $t^{*}=1 / t$, the point $t^{*}=0$ is singular). Solutions of the linear ordinary differential equation $L u=0$ are analytic in $\mathbb{C} P^{1} \backslash \Sigma_{L}$, multivalued functions. In particular, if all coefficients $a_{k}$ are constant, then any solution is a quasipolynomial $\Sigma_{j} p_{j}(t) \exp \lambda_{j} t$ for $p_{j} \in \mathbb{C}[t], \lambda_{j} \in \mathbb{C}$. There are many known results concerning the number and position of zeros of quasipolynomials, starting from the Descartes rule; see, for example, [4] for the real case when $p_{k} \in \mathbb{R}[t]$ and $\lambda_{j} \in \mathbb{R}$, and $[\mathbf{8}$, 9] for the general complex case when both the exponents $\lambda_{j}$ and the coefficients of the polynomials $p_{j}$ can be nonreal. Results concerning equations with variable coefficients are less known. Our primary objective is to generalize the results from $[\mathbf{2}, \mathbf{3}]$ and $[\mathbf{5}$, 6] to the complex case.

Notation. Throughout this paper we use the following notions and notation. If $A \subset B$ is a pair of subsets of the complex plane $\mathbb{C}$, then $A \Subset B$ means that the distance between $A$ and $\mathbb{C} \backslash B$ is strictly positive. We denote this distance (the width of the gap $B \backslash A)$ by $\operatorname{gap}(A, B)$.

We call a bounded subset $A \subset \mathbb{C}$ a closed curvilinear polygon (in short, a closed CP-gon), if $A$ is the closure of its interior $\breve{A}$, and this interior is an open connected

Received 20 January 1995

1991 Mathematics Sublect Classification 34A20.

J. London Math. Soc. (2) 56 (1997) 305-319 
simply connected subset (topological disk) whose boundary is piecewise smooth (hence of finite length). An open CP-gon is the interior of a closed CP-gon.

The length of a piecewise smooth curve $\Gamma$ is denoted by $\ell(\Gamma)$. We say that $K \Subset U$ is a (properly) nested pair, if $K \Subset U$ are CP-gons (and, of course, $\operatorname{gap}(K, U)>0$ ).

For any open bounded set $U$ denote by $\mathscr{A}(U)$ the space of functions holomorphic in some open neighbourhood of the closure $\bar{U}$. For a function $f \in \mathscr{A}(U)$ we denote $\mathbf{M}_{U}(f)=\sup _{t \in U}|f(t)|=\max _{t \in \hat{O} U}|f(t)|$.

Finally, we put $\ln _{+} x=\max (\ln x, 0)$.

Let $K \Subset \mathbb{R}$ be a compact real segment and $U$ an open CP-gon such that $K \Subset U \Subset \mathbb{C} \backslash \Sigma_{L}$. Assume that $L$ is an operator with real rational coefficients $a_{k}(t) \in \mathbb{R}(t)$, and denote by $\|L\|_{U}$ the magnitude of the coefficients of the operator $L$ :

$$
\|L\|_{U}=\max _{k=1, \ldots, n} \mathbf{M}_{U}\left(a_{k}\right)=\max _{\substack{k=1, \ldots, n, t \in \in U}}\left|a_{k}(t)\right| .
$$

In [2] it was proved that there exist a constant $\beta=\beta(K, U)$ depending only on the relative positions of the sets $K$ and $U$, such that the number of isolated zeros in $K$ of any solution of the equation $L u=0$, is at most $\beta \cdot\left(\|L\|_{U}+n\right)$, provided that $u$ is real on $K$. In this paper we show that this result in fact holds without any realness assumption.

Theorem 1. Let $K \Subset U$ be two CP-gons. Then for any linear differential operator $L=\partial^{n}+\sum_{k=1}^{n} a_{k}(t) \partial^{n-k}$ with $a_{k} \in \mathscr{A}(U)$, there exists a constant $\beta=\beta(K, U)$ such that any solution of the equation $L u=0$ can have at most $\beta \cdot\left(\|L\|_{U}+n \ln n\right)$ complex isolated zeros in $K$.

\subsection{Polynomial envelopes of linear differential operators}

As before let $L u=0$ be a linear ordinary differential equation with rational coefficients. Choose an arbitrary fundamental set of solutions $f_{1}(t), \ldots, f_{n}(t)$ for this equation, and let $d$ be a natural number. The polynomial $d$-envelope of the operator $L$ is defined as the linear space of combinations of the form

$$
\sum_{j=1}^{n} \sum_{k=0}^{n-1} p_{j k}(t) f_{j}^{(k)}(t) \quad \text { for } p_{j k} \in \mathbb{C}[t], \operatorname{deg} p_{j k} \leqslant d
$$

There are several situations when polynomial envelopes appear naturally. It can be shown that any complete abelian integral (that is, the integral of a polynomial 1-form $\omega=P(x, y) d x+Q(x, y) d y$ over closed level curves of another polynomial $H=H(x, y))$, after multiplication by an appropriate polynomial, belongs to the polynomial $d$-envelope of a certain Fuchsian differential operator with $d=O(\operatorname{deg} \omega)$; see $[\mathbf{1 0}]$.

Another situation in which the polynomial envelopes appear is iteration of differential operators. Let $f(t)$ be any function analytic in some open neighbourhood of a compact set $K$. Let $n_{k}$ be the number of isolated zeros of the $k$ th derivative $f^{(k)}(t)$ in $K$. How rapidly can the sequence $n_{k}$ grow as $k \rightarrow \infty$ ? If the function $f$ were a 
solution to some linear differential equation $L f=0$ of order $n$ with rational coefficients, then clearly

$$
f^{(k)}=\sum_{l=0}^{n-1} r_{k l} f^{(\ell)} \text { for } r_{k \ell} \in \mathbb{C}(t)
$$

The same happens if, instead of iterating the operator $\partial$, we take any other differential operator $D$ with rational coefficients. After getting rid of the common denominators of all coefficients $r_{k l}$ one arrives at an element from the polynomial envelope (the corresponding order $d$ is yet to be determined) of $L$.

One of the main problems considered in this paper is the problem of distribution of zeros in polynomial envelopes. More precisely, we fix the operator $L$ and a compact subset $K \Subset \mathbb{C} P^{1} \backslash \Sigma_{L}$ and try to place an upper bound on the maximal number of complex isolated zeros, which an arbitrary function from the polynomial $d$ envelope of $L$ may have in $K$ as $d \rightarrow \infty$.

It appears that the growth of the number of zeros in polynomial envelopes depends on the choice of the operator $L$. Indeed, if $L$ is an equation with constant coefficients and all the roots of the characteristic equation are real, then on any real segment $K$ a real function from the polynomial $d$-envelope can have at most $O(d)$ zeros (since such a function is a quasipolynomial).

A linear differential equation $L u=0$ with meromorphic coefficients defines a complex linear representation of the fundamental group $\pi_{1}\left(\mathbb{C} \backslash \Sigma_{L}\right)$ in the linear space of solutions of that equation (given by analytic continuation of solutions). The equation is said to be irreducible if this representation is irreducible. For Fuchsian equations there exists an equivalent algebraic condition: $L$ cannot be represented as a nontrivial composition of two differential operators of strictly lower order with rational coefficients, see [6].

If $L$ is an irreducible operator with real coefficients, then it was proved in [3] that for any compact real segment $K \in \mathbb{R} \backslash \Sigma_{L}$ the number of zeros in the polynomial $d$ envelope is at most double exponential in $d$ as $d \rightarrow \infty$ : this number is at most $\exp \exp \tilde{c} d$, where $\tilde{c}=\tilde{c}(K, L)<\infty$ is a constant depending only on $K$ and $L$. Later in $[\mathbf{5 , 6}]$ this estimate was improved to a simple exponential in $d$ (as in Theorem 2 below but for the real case only).

A. Khovanski1, extending the ideas of G. Petrov [7], proved quite recently that for an arbitrary Fuchsian equation with only real characteristic exponents at every singular point of $L$, the number of isolated zeros in the polynomial $d$-envelope of $L$ is at most linear in $d$ on any segment $K$, provided that we consider only functions which are real on $K$ (unpublished).

Our principal result is the following theorem, which gives a much weaker estimate, but without any assumption on the types of singular points.

THEOREM 2. If the operator L is irreducible, then for any compact subset $K \Subset \mathbb{C} P^{1} \backslash \Sigma_{L}$ there exists a finite number $c=c(K, L)$, depending only on $K$ and $L$, such that any function from the polynomial d-envelope of $L$ can have at most $\exp (c d)$ complex isolated zeros in $K$ as $d \rightarrow \infty$.

As a corollary, we obtain an upper bound for the number of zeros of successive derivatives of functions defined by irreducible differential equations. 
THEOREM 3. If a function $f$ satisfies an irreducible equation $L u=0$ with rational coefficients, then for any subset $K \Subset \mathbb{C} P^{1} \backslash \Sigma_{L}$ there exists a finite constant $c^{\prime}=$ $c^{\prime}(K, L)$ such that the derivative $f_{d}=f^{(d)}(t)$ can have at most $\exp \left(c^{\prime} d\right)$ complex isolated zeros on $K$ for all large natural $d$.

\subsection{Complex Rolle theorem}

The proof of the simple exponential estimate in [6] is based on a number of arguments, some of them complex analytic, others completely real. Among the latter, the Rolle theorem is an important ingredient of the proof.

Let $K$ be a real interval or segment and $f: K \rightarrow \mathbb{R}$ a real analytic function. Denote by $N_{K}(f)$ the number of isolated zeros of $f$ on $K$, counted with their multiplicities. Then the Rolle theorem asserts that

$$
N_{K}\left(f^{\prime}\right) \geqslant N_{K}(f)-1 \text {. }
$$

If $f=u / v$ is a meromorphic function on $K$ (the ratio of two real analytic functions), then the Rolle theorem in the above form can be applied to each interval of continuity of $f$, thus yielding the inequality

$$
N_{K}\left(f^{\prime}\right) \geqslant N_{K}(f)-N_{K}(v)-1 .
$$

If $f, g: K \rightarrow \mathbb{R}$ are real analytic functions, then the following trivial equality holds:

$$
N_{K}(f g)=N_{K}(f)+N_{K}(g) .
$$

Clearly, the first two inequalities (related to the Rolle theorem) do not admit a straightforward generalization for nonreal functions. In [8,9] M. Voorhoeve found a very elegant generalization (or rather a substitute) for the number of isolated zeros of

$f$ in $K$ : he constructed an index $\mathbf{V}_{\Gamma}(f)$ which behaves very much like the number of zeros, but for which the Rolle inequality holds despite the fact that $f$ is no longer realvalued. More precisely, instead of the number of complex isolated zeros, which is equal to the index $\oint_{\Gamma} d / d t \operatorname{Arg} f(t) d t$ on the boundary $\Gamma$ of an infinitesimally thin neighbourhood of $K=[\alpha, \beta]$, Voorhoeve considers the total variation of the argument function,

$$
\mathbf{V}_{\Gamma}(f)=\oint_{\Gamma}\left|\frac{d}{d t} \operatorname{Arg} f(t)\right| d t\left|=2 \int_{\alpha}^{\beta}\right| \operatorname{Im}\left(f^{\prime} / f\right) \mid d t .
$$

Then $\mathbf{V}_{\Gamma}\left(f^{\prime}\right) \geqslant \mathbf{V}_{\Gamma}(f)-\pi$ and $\left|\mathbf{V}_{\Gamma}(f)-\mathbf{V}_{\Gamma}(g)\right| \leqslant \mathbf{V}_{\Gamma}(f g) \leqslant \mathbf{V}_{\Gamma}(f)+\mathbf{V}_{\Gamma}(g)$, see [8]. These results were then applied to an investigation of the distribution of zeros of quasipolynomials without any realness assumption.

However, the index $\mathbf{V}_{\Gamma}(f)$ cannot easily be computed for classes of functions $f$ other than quasipolynomials and for boundaries of general domains in $\mathbb{C}$.

Now let $K$ be a closed CP-gon with boundary $\Gamma=\partial K$ and suppose that $f \in \mathscr{A}(K)$. We construct in $\S 2$ below another index, similar to the number $N_{K}(\cdot)$, which is well defined for complex valued functions and which will exhibit a similar behaviour. This index requires not one but rather two sets.

Let $U \subset \mathbb{C}$ be an open CP-gon with boundary $\Gamma$, and $K \Subset U$ a compact subset of $U$ (at a positive distance from $\Gamma$ ). 
Definition. The Bernstein index of an analytic function $f \in \mathscr{A}(U)$ with respect to the pair of sets $K \Subset U$, is the number

$$
\mathbf{5}_{K, U}(f)=\ln \frac{\mathbf{M}_{U}(f)}{\mathbf{M}_{K}(f)} \geqslant 0
$$

(recall that $\mathbf{M}_{X}(f)$ is the maximum modulus of $f$ on the closure of $\left.\bar{X}\right)$. For $f \equiv 0$ we put $\mathbf{5}_{K, U}(f)=0$ by definition.

This index (modulo a constant factor depending only on the geometry of the pair) yields an upper estimate for the number of complex isolated zeros of $f$ in $K$, as the following simple result shows.

Generalized JenSEN INEQUALITY [2]. For any compact subset $K \Subset U$ of a CP-gon $U$ there exists a finite number $\gamma=\gamma(K, U)<\infty$ such that the number $N_{K}(f)$ of complex isolated zeros of $f$ in $K$ admits an upper estimate of the form

$$
\forall f \in \mathscr{A}(U) \quad N_{K}(f) \leqslant \gamma(K, U) \mathbf{5}_{K, U}(f) .
$$

The Bernstein index can be considered as a reasonable substitute for the number of complex zeros or the degree of a polynomial (in fact, for polynomials the Bernstein index is asymptotically equivalent to the degree, see below). In particular, the Bernstein index is 'almost additive' with respect to multiplication. On the other hand, the following easy statement (proved in §2) shows that unlike the index $N_{K}(\cdot)$, the Bernstein index of a function and its derivative are related by an inequality similar to (1.1).

THEOREM 4 (Generalized Rolle theorem). Let $K \Subset U$ be a pair of CP-gons (hence the interior $\breve{K}$ is nonempty) and let $K^{\prime} \Subset K$ be a closed $C P$-gon with gap $\left(K^{\prime}, K\right)>0$. Then there exists a constant $\rho=\rho\left(K^{\prime}, K, U\right)<\infty$ such that

$$
\forall f \in \mathscr{A}(U) \quad \mathbf{5}_{K^{\prime}, U}\left(f^{\prime}\right) \geqslant \mathbf{5}_{K, U}(f)-\rho\left(K^{\prime}, K, U\right) .
$$

The constant $\rho$ can be described in geometric terms as follows:

$$
\rho\left(K^{\prime}, K, U\right) \leqslant 1+\ln _{+} \ell(\partial K)+\ln _{+} \ell(\partial U)+2 \ln _{+}\left(\operatorname{gap}\left(K^{\prime}, K\right)\right)^{-1} .
$$

The property (1.2) can also be generalized for the Bernstein index. All these results are gathered in $\S 2$ below.

\subsection{Further generalizations}

Theorems 1 and 2, as they are formulated above, are the simplest versions; in fact, many assumptions may be essentially relaxed. For functions determined by differential equations, one may allow for singularities of the equation, provided that the solution $f(t)$ is analytic. The same arguments as in [2] allow us to deduce the following result from Theorem 1 . 
THEOREM 1'. Let $K \Subset U$ be a pair of sets, as in Theorem 1, and let

$$
L=a_{0}(t) \partial^{n}+a_{1}(t) \partial^{n-1}+\cdots+a_{n-1}(t) \partial+a_{n}(t)
$$

be a differential operator with analytic coefficients $a_{k} \in \mathscr{A}(U)$, so that $\Sigma_{L}=\left\{a_{0}(t)=0\right\}$. Assume that $\mathbf{M}_{K}\left(a_{0}\right) \geqslant 1$ (one may always achieve this by rescaling $L \mapsto \mu L$ for $\mu \in \mathbb{C}$ ). Then any analytic solution $f \in \mathscr{A}(U)$ of the equation $L u=0$ can have at most $\left(\|L\|_{U}+n\right)^{v}$ complex isolated zeros in $K$, where $v=v(K, U)$ is a finite constant depending only on the pair $K, U$.

The second result also admits a generalization. Let $U \subseteq \mathbb{C}$ be an arbitrary open set with fundamental group $G=\pi_{1}(U, \bullet)$, and $\mathcal{O}(U)$ the ring of functions holomorphic (and single-valued) in $U$ : for functions from $\mathcal{O}(U)$ we do not specify any boundary behaviour. Then the noncommutative ring $\mathscr{D}(U)=\mathcal{O}(U)[\partial]$ of differential operators with coefficients in $\mathcal{O}(U)$ is defined in the natural way. A complex vector representation of the fundamental group $G$ can be associated with each operator, and an operator $L \in \mathscr{D}(U)$ is said to be irreducible if this representation is irreducible. In the same way as before, the polynomial envelope of $L$ can be defined.

THEOREM 2'. If $L$ is a unitary irreducible operator in $\mathscr{D}(U)$ and $K \Subset U$ an arbitrary compact set, then the number of isolated zeros of any function from the polynomial $d$ envelope of $L$ in $K$ can be at most $\exp (c d)$, where $c(K, L, U)<\infty$ is a finite constant.

Reduction from Theorem 2 to Theorem $2^{\prime}$ for the real case was explained in [6]; the same arguments (but with Theorem 4 replacing the Rolle lemma) also prove the complex result.

In $[5,6]$ it was noted that the irreducibility condition can be relaxed to a certain extent. This remark remains valid for the complex case as well; and the proof from [6], after obvious modifications, establishes the following result. An operator $L \in \mathbb{C}(t)[\partial]$ is said to be essentially irreducible [6], if it can be factored (in this ring) as

$$
L=L^{*} \circ P_{k} \circ P_{k-1} \circ \cdots \circ P_{2} \circ P_{1} \text { for } P_{i} \in \mathbb{C}(t)[\partial] \text { with ord } P_{i}=1,
$$

and the factor $L^{*}$ is irreducible in the original sense.

THEOREM 3'. If $f$ satisfies the equation $L f=0$, where the operator $L \in \mathbb{C}(t)[\partial]$ has rational coefficients, then for any compact $K \Subset \mathbb{C} \backslash \Sigma_{L}$ we have

$$
N_{K}\left(f^{(d)}\right) \leqslant \exp \left(c^{\prime} d\right) \quad \text { for some } c^{\prime}=c^{\prime}(K, L)<\infty .
$$

\section{Bernstein index and a complex analogue of the Rolle theorem}

In this section we develop the theory of the Bernstein index and prove the complex Rolle theorem in general settings. As already noted in $\S 1$, this index is a generalization of the degree of a polynomial. The choice of the term 'Bernstein index' is motivated by the following form of the Bernstein inequality.

BERNSTEIN INEQUALITY (see $[\mathbf{1}, \mathrm{p} .113])$. Let $K=[-1,1]$ be the standard segment and $U=\{t \in \mathbb{C} ;|t-1|+|t+1| \leqslant 2 R\}$ for $R>1$, the ellipse with foci at \pm 1 . Then for any polynomial $p$ of degree $d$ one has $\mathbf{b}_{K, U}(p) \leqslant d \cdot \ln R$, and this bound is sharp. 
From the definition of $\boldsymbol{b}_{K, U}(\cdot)$ it follows immediately that this index is a zero order homogeneous functional: $\boldsymbol{\zeta}_{K, U}(\lambda f)=\mathrm{\zeta}_{K, U}(f)$ for any $f$ and any complex $\lambda \neq 0$. Thus without loss of generality we may rescale any function by multiplying it by an appropriate constant.

\subsection{Lower bounds for analytic functions}

Let $Z \subset \mathbb{C}$ be an arbitrary bounded subset. Consider all finite coverings of $Z$ by discs, and denote by $|Z|$ the minimal sum of diameters of disks (the minimum, or rather infimum, is taken over all finite coverings). Let $K \subseteq U$ be a compact subset of an open CP-gon $U$, and $f \in \mathscr{A}(U)$ a nonzero analytic function. Without loss of generality we may rescale $f$ in such a way that $\mathbf{M}_{U}(f)=1$. For any $\varepsilon>0$, consider the set $Z_{\varepsilon}(f)=\{t \in U:|f(t)| \leqslant \varepsilon\}$ : then $Z_{0}(f)$ is a finite union of points, hence $\left|Z_{0}(f)\right|=0$, and $Z_{1}(f)=U$.

Lemma 1 [3]. For any open $C P$-gon $U$ and any $K \Subset U$ there exist two finite constants $\alpha=\alpha(K, U), \beta=\beta(K, U)$ depending only on the pair $K \Subset U$ such that for any function $f \in \mathscr{A}(U)$ and any positive $h>0$ one can find a finite union $D_{h}$ of disks with the sum of diameters less than $h$ so that

$$
\min _{t \in K \backslash D_{h}}|f(t)| \geqslant\left(\frac{\mathbf{M}_{K}(f)}{\mathbf{M}_{U}(f)}\right)^{\alpha+\beta \mathrm{nn}_{+}(1 / h)} .
$$

COROllary. For any pair $K \Subset U$ as above, there exists a pair of finite constants $C=C(K, U)$ and $v=v(K, U)$, depending only on the geometry of the pair, such that

$$
\forall f \in \mathscr{A}(U) \quad\left|Z_{\varepsilon}(f) \cap K\right| \leqslant C \varepsilon^{(1 / v d)} \quad \text { for } d=\mathrm{Б}_{K, U}(f) .
$$

Proof of the Corollary. Expressing $h$ in terms of $\varepsilon$ from the inequality (2.1) and substituting $\mathbf{M}_{U}(f) / \mathbf{M}_{K}(f)=\exp d$, we arrive at the required form.

REMARK. If $f$ has a zero of multiplicity $d$ at the origin, then locally the set of those $t$ for which $|f(t)| \leqslant \varepsilon$ is a slightly distorted disc of diameter $C \varepsilon^{1 / d}$. The inequality $\left(2.1^{*}\right)$ is a globalization of this observation. The upper bound for $\left|Z_{\varepsilon}(f)\right|$ means that an analytic function with a bounded Bernstein index cannot be uniformly too small on 'large' subsets of $K$.

\subsection{Properties of the Bernstein index}

By definition, the Bernstein index (as a functional $\mathscr{A}(U) \rightarrow \mathbb{R}$ ) depends on the choice of the nested pair $(K, U)$. This dependence is monotonic: if $K^{\prime} \subset K$ and $U^{\prime} \subset U$, then

$$
\mathbf{5}_{K^{\prime}, U}(\cdot) \geqslant \mathbf{5}_{K, U}(\cdot) \geqslant \mathbf{5}_{K, U}(\cdot) .
$$

The following result indicates that the Bernstein index, when considered modulo a finite factor, is independent of the choice of the inner compactum $K$ provided that $|K|>0$. 
Lemma 2. If $K_{i} \Subset U$ for $i=1,2$, are two nested pairs, and $\left|K_{i}\right|>0$, then the corresponding Bernstein indices are equivalent as functionals on $\mathscr{A}(U)$ : there exists a finite strictly positive constant $c$ depending only on the geometry of the sets $K_{1}, K_{2}, U$, such that

$$
c \cdot \mathbf{b}_{K_{1}, U}(\cdot) \leqslant \mathbf{b}_{K_{2}, U}(\cdot) \leqslant \frac{1}{c} \cdot \mathbf{b}_{K_{1}, U}(\cdot)
$$

Proof. Let $K=K_{1} \cup K_{2}$. Then clearly $K \Subset U$, and for an arbitrary $f \in \mathscr{A}(U)$ with $\mathbf{M}_{U}(f)=1$ we have $d_{i}=\mathbf{5}_{K_{i}, U}(f) \geqslant d=\mathbf{5}_{K, U}(f)$. Then for $\varepsilon=\frac{1}{2}\left|K_{2}\right|$, any finite union of disks with the sum of diameters less than $\varepsilon$ cannot cover $K_{2}$, so by Lemma 1 the inequality $\mathbf{M}_{K_{2}}(f) \geqslant \mathbf{M}_{K}(f)^{\alpha+\beta \ln 1 / \varepsilon}$ holds; see (2.1). Since $\mathbf{M}_{K}(f)=\exp (-d)$, we have $\mathbf{M}_{K_{2}}(f) \geqslant \exp (-c d)$, where $c=\alpha+\beta \ln \left(1 /\left|K_{2}\right|\right)+\ln 2$, so finally

$$
d_{2} \leqslant \ln \frac{1}{\exp (-c d)}=c d \leqslant c d_{1}
$$

Later on we shall always implicitly assume (unless stated otherwise) that all nested pairs $K \Subset U$ are pairs of CP-gons; the condition $|K|>0$ will then be automatically satisfied. Since we have freedom in the choice of $K$, this is not a serious restriction.

\subsection{Complex Rolle theorem in the simplest case: demonstration of Theorem 4}

As usual, we consider an arbitrary $f \in \mathscr{A}(U)$, this time scaled so that $\mathbf{M}_{K}(f)=1$. Take a simple contour $\Gamma=\partial K$ encircling $K^{\prime}$ : clearly, $\operatorname{dist}\left(K^{\prime}, \Gamma\right) \geqslant \operatorname{gap}\left(K^{\prime}, K\right)$. Then by the Cauchy integral formula, for any point $t \in K^{\prime}$ we have

$$
\left|f^{\prime}(t)\right|=\left|\frac{1}{2 \pi \sqrt{ }(-1)} \oint_{\Gamma} \frac{f(\zeta) d \zeta}{(t-z)^{2}}\right| \leqslant \frac{\mathbf{M}_{K}(f) \cdot \ell(\Gamma)}{2 \pi \operatorname{gap}^{2}\left(K^{\prime}, K\right)} \leqslant \frac{1}{2} \ell(\partial K) \cdot \operatorname{gap}^{-2}\left(K^{\prime}, K\right) .
$$

On the other hand, if we take any path $\Gamma^{\prime}$ connecting $K$ and $\hat{\Gamma}=\partial U$ (the length of such path can be made less than $\ell(\partial U))$ and integrate the differential form $d f(t)=$ $f^{\prime}(t) d t$ along the union of two $\operatorname{arcs} \Gamma^{\prime} \cup \hat{\Gamma}$, then (since the maximum of $\mathbf{M}_{U}(f)$ is achieved somewhere on $\hat{\Gamma}$ ), we have

$$
\mathbf{M}_{U}(f) \leqslant \mathbf{M}_{K}(f)+\left(\ell\left(\Gamma^{\prime}\right)+\ell(\hat{\Gamma})\right) \cdot \mathbf{M}_{U}\left(f^{\prime}\right) \leqslant 1+2 \ell(\partial U) \cdot \mathbf{M}_{U}\left(f^{\prime}\right) .
$$

From this inequality we conclude (since $\exp x-1 \geqslant \exp (x-1)$ for all $x \geqslant 1)$ that

$$
\mathbf{5}_{K^{\prime}, U}\left(f^{\prime}\right)=\ln \frac{\mathbf{M}_{U}\left(f^{\prime}\right)}{\mathbf{M}_{K^{\prime}}\left(f^{\prime}\right)} \geqslant \ln \left(\frac{\operatorname{gap}^{2}\left(K^{\prime}, K\right)\left(\exp \mathbf{5}_{K, U}(f)-1\right)}{\ell(\partial K) \ell(\partial U)}\right) \geqslant \mathbf{5}_{K, U}(f)-\rho\left(K^{\prime}, K, U\right),
$$

if we put $\rho=1+2 \ln _{+} \operatorname{gap}^{-1}\left(K^{\prime}, K\right)+\ln _{+} \ell(\partial K)+\ln _{+} \ell(\partial U)$. This proves the inequality (1.5) for all $f$ with $d=\mathbf{5}_{K, U}(f) \geqslant 1$. For $d<1$ the inequality is valid automatically, since $\mathbf{b}_{K^{\prime}, U}\left(f^{\prime}\right)$ is always nonnegative.

REMARK. All inequalities concerning the Bernstein index are meaningful for large values of that index. Thus we shall not discuss specially the case of small $d$ any further. 


\subsection{Bernstein index of a product: subadditivity}

Let $f_{1}, \ldots, f_{n} \in \mathscr{A}(U)$ be analytic functions and $v_{1}, \ldots, v_{n}$ natural numbers.

Lemma 3. For any nested pair $K \Subset U$ with $|K|>0$ there exist two finite numbers $a(K, U), b(K, U)$ such that for any collection of $f_{j}$ and $v_{j} \in \mathbb{N}$ we have

$$
\mathbf{5}_{K, U}\left(f_{1}^{v_{1}} \cdots f_{n}^{v_{n}}\right) \leqslant(a+b \ln n) \sum_{j=1}^{n} v_{j} \mathbf{5}_{K, U}\left(f_{j}\right)
$$

Proof. Set $d_{i}=\mathbf{Б}_{K, U}\left(f_{i}\right)$, assume that $\mathbf{M}_{U}\left(f_{i}\right)=1$ and let $f=\Pi_{j} f_{j}^{v_{j}}$. Take any positive $\varepsilon<|K| / n$ and apply the basic lower estimate (2.1) to each function; our scaling condition implies then that there exists a point $t \in K$, at which all lower estimates of the type (2.1) for all $f_{i}$ hold simultaneously, hence

$$
\mathbf{M}_{K}(f) \geqslant \prod_{j} \mathbf{M}_{K}\left(f^{v_{j}}\right)^{\alpha+\beta \ln n-\ln |K|} \geqslant \prod_{j} \exp \left(v_{j} d_{j}(a+b \ln n)\right),
$$

if we put $a=\alpha-\ln |K|, b=\beta$. But since $\mathbf{M}_{U}(f) \leqslant \Pi_{j} \mathbf{M}_{U}\left(f_{j}\right)=1$, we have immediately the upper estimate for $\mathbf{b}_{K, U}(f)$.

Corollary. $\forall f, g \in \mathscr{A}(U), \mathbf{5}_{K, U}(f g) \leqslant \operatorname{const}(K, U)\left(\mathbf{Б}_{K, U}(f)+\mathbf{5}_{K, U}(g)\right)$.

\subsection{Multiplication and Bernstein index}

The previous assertion gives an upper estimate for the Bernstein index of the product of two functions. The assertion of the corollary is symmetric with respect to $f$ and $g$. However, in order to formulate the inverse inequality (the lower bound for the Bernstein index of a product) it is natural to consider the 'differential operator' $R_{g}: f \mapsto g \cdot f$ of zero order.

Assume that the function $g$ is analytic in the closure of a larger $\mathrm{CP}$-gon $V \subset \mathbb{C}$ : $g \in \mathscr{A}(V)$ and $U \Subset V$.

Lemma 4. Let $U^{\prime} \Subset U$ be an intermediate CP-gon: $K \Subset U^{\prime} \Subset U$. Then there exists a constant $\theta=\theta\left(U^{\prime}, U, V\right)<\infty$ such that

$$
\forall f \in \mathscr{A}(U) \quad \mathbf{5}_{K, U}(f g) \geqslant \mathbf{5}_{K, U^{\prime}}(f)-\theta\left(U^{\prime}, U, V\right) \mathbf{5}_{U, V}(g) .
$$

The constant $\theta=\theta\left(U^{\prime}, U, V\right)$ admits the following upper estimate:

$$
\theta\left(U^{\prime}, U, V\right) \leqslant \alpha(U, V)+\beta(U, V) \ln _{+}\left(\operatorname{gap}^{-1}\left(U^{\prime}, U\right)\right) .
$$

The proof is based on the following simple geometrical argument.

Lemma 5 (Thick belt lemma). Suppose that $K \Subset U$ is a pair of closed CP-gons and $\operatorname{gap}(K, U)=h$. Then for any set $Z \subset \mathbb{C}$ with $|Z|<h / 2$ one can construct a closed simple 
contour (a topological circle) $\Gamma \subset U$ such that $K$ lies inside the closure of the domain bounded by $\Gamma$ and $\Gamma \cap Z=\varnothing$. Moreover, $\Gamma$ can be chosen piecewise smooth and $\ell(\Gamma)$ $\leqslant \ell(\partial U)+\pi h$.

In other words, there exists an intermediate CP-gon $V$ with $K \subset V \subset U$, whose border $\Gamma=\partial V$ is disjoint with $Z$.

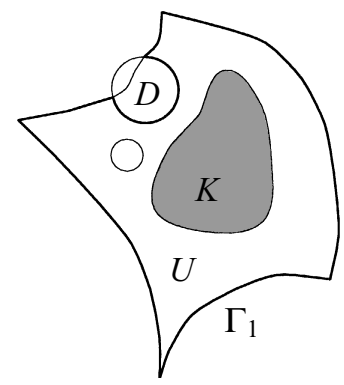

(a)

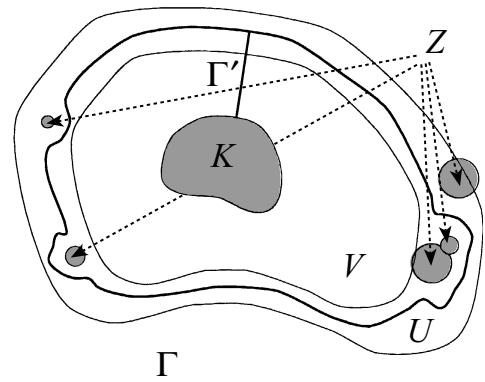

(b)

FIG. 1. (a) Avoiding one disk $D$.

(b) Construction of a path inside the thick belt (see the proof of Lemma 5).

Proof of Lemma 5. It is sufficient to prove this lemma when $Z$ is a finite union of disks with the sum of diameters less than $3 h / 4$. Set $\Gamma_{0}=\partial U$. The proof goes by induction in the number of disks.

If there is only one disk $D$, then either $\partial D \cap \Gamma_{0}=\varnothing$ and hence $\Gamma_{0}$ satisfies all requirements and there is nothing to prove, or one may take the concentric disk $D^{\prime}$ of radius arbitrarily close to that of $D$, and this will intersect $\Gamma_{0}$ transversally; hence the boundary $\Gamma_{1}$ of the union $(\mathbb{C} \backslash U) \cup D^{\prime}$ will be piecewise-smooth. Clearly, the distance from $\Gamma_{1}$ to $K$ will be arbitrarily close to $h-\operatorname{diam} D^{\prime}$ and

$$
\ell\left(\Gamma_{1}\right) \leqslant \ell\left(\Gamma_{0}\right)+\pi \operatorname{diam} D^{\prime} .
$$

Iterating these arguments, we arrive at the assertion of the lemma.

Remark. Assume that $K$ contains a disk $D$ of diameter $h>0$. Then one can, in addition to the contour $\Gamma$, construct a path (a simple arc) $\Gamma^{\prime}$ connecting $K$ with $\Gamma$ and avoiding $Z$; the length of this arc will be at most $\frac{1}{2} \ell(\partial U)$.

Indeed, take all rays beginning at all points of the small disk $D$ and parallel to some direction in the plane. Then all of these rays will meet the boundary $\partial U$ and hence the contour $\Gamma$, and the distance from the beginning of each ray to the point of intersection will be at most $\frac{1}{2} \ell(\partial U)$. Since $|Z|<h$, not all rays can intersect $Z$, hence there exists a segment $\Gamma^{\prime}$ with one endpoint in $D$, another endpoint on $\Gamma$ and avoiding $Z$.

Proof of Lemma 4. Without loss of generality we may assume that $f$ and $g$ are scaled so that $\mathbf{M}_{K}(f)=1$ and $\mathbf{M}_{U}(g)=1$ respectively. By the basic lower estimate (2.1), one may choose a set $Z \subset V$ with $|Z|<\varepsilon=\operatorname{gap}\left(U^{\prime}, U\right)$ such that outside this set the multiplier $g$ admits the lower estimate:

$$
\begin{gathered}
\forall t \in U \backslash Z \quad|g(t)| \geqslant \exp \left(-\theta \mathbf{b}_{U, V}(g)\right), \\
\theta=\theta\left(U^{\prime}, U, V\right) \leqslant \alpha+\beta \cdot \ln _{+} \operatorname{gap}^{-1}\left(U^{\prime}, U\right) \quad \text { with } \alpha=\alpha(U, V), \beta=\beta(U, V) .
\end{gathered}
$$



Then

Using the thick belt lemma, choose a contour $\Gamma$ in the belt $\bar{U} \backslash U^{\prime}$, avoiding $Z$.

$$
\mathbf{M}_{K}(f g) \leqslant \mathbf{M}_{K}(f) \cdot \mathbf{M}_{K}(g) \leqslant \mathbf{M}_{K}(f) \cdot \mathbf{M}_{U}(g)=1,
$$

and on the other hand, using the maximum modulus principle, we see that

$$
\begin{aligned}
\mathbf{M}_{U}(f g) & \geqslant \mathbf{M}_{\Gamma}(f g) \geqslant \mathbf{M}_{\Gamma}(f) \cdot \min _{t \in \Gamma}|g(t)| \\
& \geqslant \mathbf{M}_{U^{\prime}}(f) \cdot \min _{t \in \Gamma}|g(t)|=\exp \mathbf{5}_{K, U^{\prime}}(f) \cdot \exp \left(-\theta \mathbf{5}_{U, V}(g)\right) .
\end{aligned}
$$

From these estimates it follows immediately that

$$
\mathbf{5}_{K, U}(f g) \geqslant \mathbf{5}_{K, U}(f)-\theta \mathbf{5}_{U, V}(g) .
$$

REMARK. If $p \in \mathbb{C}[t]$ is a polynomial of degree $n$, then the above arguments can be simplified, since instead of the lower bound (2.1) one may apply the Cartan inequality directly; see [2]. As a result, we can simplify the final inequality:

$$
\mathbf{5}_{K, U}(f p) \geqslant \mathbf{5}_{K, U^{\prime}}(f)-\Theta n, \quad \Theta=\Theta\left(U, U^{\prime}\right)=1+\ln _{+} \operatorname{gap}^{-1}\left(U, U^{\prime}\right) .
$$

\subsection{Meromorphic functions and the complex Rolle theorem in the general case}

Now we can establish a complex analogue of the inequality (1.2). The Bernstein index is defined for analytic functions (without poles in $\bar{U}$ ); thus one should look for an indirect way to define $\mathbf{5}_{K, U}(f)$ for meromorphic $f$. However, we may avoid this difficulty by the following trick.

Let $f=h / g$ be a meromorphic function, $g, h \in \mathscr{A}(U)$. Then $f^{\prime}=\left(h^{\prime} g-h g^{\prime}\right) / g^{2}$, and for the number of real zeros on any real interval $K \subset \mathbb{R}$ we would have $N_{K}(f)=$ $N_{K}(h), N_{K}\left(f^{\prime}\right) \leqslant N_{K}\left(h^{\prime} g-h g^{\prime}\right)=N_{K}\left(g^{2} f^{\prime}\right)$. The inequality (1.2) would then imply the inequality

$$
N_{K}\left(h^{\prime} g-h g^{\prime}\right) \geqslant N_{K}(h)-N_{K}(g)-1 \quad \forall g, h \text { real analytic on } K .
$$

It is this form which allows for complex generalization.

THEOREM 4' (Complex Rolle theorem in the general case). Let $K^{\prime} \Subset K \Subset U^{\prime} \Subset$ $U \Subset V \subseteq \mathbb{C}$ be four nested CP-gons. Then

$$
\mathbf{5}_{K^{\prime}, U}\left(h^{\prime} g-h g^{\prime}\right) \geqslant \mathbf{5}_{K, U}(h)-\theta \cdot \mathbf{5}_{U, V}(g)-\rho \quad \forall h \in \mathscr{A}(U), \forall g \in \mathscr{A}(V) \text {. }
$$

Here $\theta, \rho<\infty$ are two constants depending only on the geometry of the sets and similar to the constants from Theorem 4 and Lemma 4 respectively:

$$
\begin{gathered}
\theta=\theta\left(U^{\prime}, U, V\right) \leqslant 2 \alpha(U, V)+2 \beta(U, V) \ln _{+}\left(\operatorname{gap}^{-1}\left(U^{\prime}, U\right)\right), \\
\rho=\rho\left(K^{\prime}, K, U\right) \leqslant 1+\ln _{+} \ell(\partial K)+\ln _{+} \ell(\partial U)+2 \ln _{+}\left(\operatorname{gap}^{-1}\left(K^{\prime}, K\right)\right) .
\end{gathered}
$$

Proof. The inequality (2.4) combines some features of both (1.5) and (2.2); to obtain a lower estimate for the Bernstein index of $h^{\prime} g-h g^{\prime}$, one pays by decreasing the size of the interior compactum and enlarging the ambient open set. The proof is also a 'linear combination' of ideas used when proving (1.5) and (2.4).

We rescale $h$ and $g$ so that $\mathbf{M}_{U}(g)=1$ and $\mathbf{M}_{K}(h)=1$ respectively, and denote by $D_{g}$ the operator $h \mapsto g h^{\prime}-g^{\prime} h$. Then by the Cauchy integral formula (cf. Theorem 4),

$$
\begin{aligned}
\mathbf{M}_{K^{\prime}}\left(D_{g} h\right) & \leqslant \mathbf{M}_{K^{\prime}}(g) \cdot \mathbf{M}_{K^{\prime}}\left(h^{\prime}\right)+\mathbf{M}_{K^{\prime}}\left(g^{\prime}\right) \cdot \mathbf{M}_{K^{\prime}}(h) \\
& \leqslant 2 \ell(\partial K) / 2 \pi \operatorname{gap}^{2}\left(K^{\prime}, K\right) \leqslant \frac{1}{2} \ell(\partial K) \cdot \operatorname{gap}^{-2}\left(K^{\prime}, K\right),
\end{aligned}
$$

since $\mathbf{M}_{K}(g), \mathbf{M}_{K}(h) \leqslant 1$. 
To estimate $\mathbf{M}_{U}\left(D_{g} h\right)$ from below, we apply once again the thick belt lemma to the pair $U^{\prime}, U$ and construct a connected path $\Gamma=\Gamma^{\prime} \cup \hat{\Gamma}$ consisting of two parts: a topological circle (contour) $\Gamma^{\prime} \Subset U \backslash U^{\prime}$ and an arc (segment) $\hat{\Gamma}$ connecting $K^{\prime}$ with $\Gamma^{\prime}$ in such a way (see the remark after the thick belt lemma) that

$$
\mathbf{M}_{\Gamma}\left(g^{-1}\right) \leqslant A=\exp \left(\theta \mathbf{5}_{U, V}(g)\right),
$$

where $\theta=\theta\left(U^{\prime}, U, V\right) \leqslant \alpha(U, V)+\beta(U, V) \cdot \ln _{+} \operatorname{gap}^{-1}\left(U^{\prime}, U\right)$ and $\ell(\Gamma) \leqslant 2 \ell(\partial U)$. Along this path $f$ is continuously differentiable (the denominator does not vanish), hence by the mean value theorem $\mathbf{M}_{\Gamma}(f) \leqslant \mathbf{M}_{K \cap \Gamma}(f)+\ell(\Gamma) \cdot \mathbf{M}_{\Gamma}\left(f^{\prime}\right)$.

Since $\mathbf{M}_{\Gamma}(g) \leqslant 1$, we have

$$
\mathbf{M}_{\Gamma}(f) \geqslant \mathbf{M}_{\Gamma}(h) \geqslant \mathbf{M}_{U^{\prime}}(h), \quad \mathbf{M}_{K \cap \Gamma}(f) \leqslant \mathbf{M}_{\Gamma}\left(g^{-1}\right) \cdot \mathbf{M}_{K}(h) \leqslant A \cdot 1=A,
$$

and so we finally obtain the inequalities

$$
\mathbf{M}_{U}\left(D_{g} h\right) \geqslant \mathbf{M}_{\Gamma}\left(D_{g} h\right) \geqslant A^{-2} \mathbf{M}_{\Gamma}\left(f^{\prime}\right) \geqslant \frac{1}{A^{2} \ell(\Gamma)} \cdot\left(\mathbf{M}_{U^{\prime}}(h)-A\right) .
$$

Taking into account the fact that $\mathbf{M}_{U^{\prime}}(h)=\exp \mathbf{5}_{K, U^{\prime}}(h), A=\exp \theta \mathbf{5}_{U, V}(g)$ and $\exp x-\exp y \geqslant \exp (x-1)$ for $x-y \geqslant 1$, we obtain

$$
\begin{aligned}
\mathbf{5}_{K^{\prime}, U}\left(D_{g} h\right) \geqslant & \ln \left(\frac{\mathbf{M}_{U^{\prime}}(h)-A}{A^{2} \ell(\partial U) \cdot \ell(\partial K) \cdot \operatorname{gap}^{-2}\left(K^{\prime}, K\right)}\right) \\
\geqslant & \mathbf{5}_{K, U^{\prime}}(h)-2\left(\alpha(U, V)+\beta(U, V) \cdot \ln _{+} \operatorname{gap}^{-1}\left(U^{\prime}, U\right)\right) \cdot \mathbf{5}_{U, V}(g) \\
& -\left(1+\ln _{+} \ell(\partial K)+\ln _{+} \ell(\partial U)+2 \ln _{+} \operatorname{gap}^{-1}\left(K^{\prime}, K\right)\right),
\end{aligned}
$$

which is the estimate we need.

3. Applications: oscillations of solutions of linear ordinary differential equations with analytic coefficients

In this section we prove the theorems from $\S 1$ concerning the number of zeros of functions defined by linear ordinary differential equations with analytic coefficients. Throughout this section we assume without loss of generality that all sets are CPgons. In fact, the proofs below reproduce essentially the proofs given in [6], except for one crucial feature: instead of the real Rolle theorem one must use its complex analogue from $\S 2$. Modulo this change, all the rest goes with only minor and evident changes, and thus the exposition below is rather schematic. We refer the reader to $[\mathbf{5}$, 6] for more details if necessary.

\subsection{Oscillation and the magnitude of coefficients: demonstration of Theorem 1}

Let $(K, U)$ be a pair of sets and $L$ a unitary linear ordinary differential operator of order $n$ with coefficients in $\mathscr{A}(U)$, that is,

$$
L=\partial^{n}+\sum_{l=1}^{n} a_{k}(t) \partial^{n-k} \quad \text { for } a_{k} \in \mathscr{A}(U) .
$$

Then any solution of the equation $L u=0$ is necessarily analytic in $U$, since $L$ has no singular points there. Recall that $\|L\|=\max _{k=1, \ldots, n} \mathbf{M}_{\bar{U}}\left(a_{k}\right)$ is the magnitude of the coefficients of the operator. 
The proof of Theorem 1 can be obtained by a small modification of the proof of [3, Theorem 1], by replacing the reference to the classical Rolle theorem by that to the complex Rolle theorem (Theorem 4). In [3] it is shown that $L f=0$ implies that for some derivative $f^{(k)}$ with $0 \leqslant k \leqslant n-1$ the Bernstein index admits the following estimate:

$$
\mathbf{5}_{K^{\dagger}, U}\left(f^{(k)}\right) \leqslant \ln n+\operatorname{const}\left(K^{\dagger}, U\right) \cdot(\|L\|+1)
$$

for any set $K^{\dagger} \supset K$. Then, iterating the complex Rolle theorem (in the form given by Theorem 4) $k$ times, one can show that $\mathrm{b}_{K, U}(f) \leqslant O(\|L\|)$ if $n$ is considered as a fixed parameter.

To obtain a more precise inequality which would take the order $n$ into account, we choose a sequence of nested CP-gons $K=K_{n} \subset K_{n-1} \subset \cdots \subset K_{1} \subset K_{0}=K^{\dagger}$ with $\operatorname{gap}\left(K_{i+1}, K_{i}\right) \geqslant \operatorname{const}(K) / n$. It is clear that all lengths $\ell\left(\partial K_{i}\right)$ are bounded by a certain constant independent of $n$, hence each $\rho_{i}=\rho\left(K_{i+1}, K_{i}, U\right)$ will be of order of magnitude const $_{1}(K, U)+$ const $_{2}(K, U) \ln n$.

Iteration of the complex Rolle theorem applied to all collections $K_{i+1} \Subset K_{i} \Subset U$ for all $i=k, k-1, \ldots, 1,0$ then gives

$$
\mathbf{5}_{K_{n}, U}(f) \leqslant \mathbf{5}_{K_{0}, U}\left(f^{(k)}\right)+\text { const }_{1}(K, U) \cdot n+\text { const }_{2}(K, U) \cdot n \ln n .
$$

As for Theorem 1', it can be reduced to Theorem 1 in the same way as in [2]: if $L=a_{0}(t) \partial^{n}+\cdots+a_{n-1}(t) \partial+a_{n}(t)$ is a non-unitary differential operator with analytic coefficients, then one can find a set $Z_{0}$ with $\left|Z_{0}\right|$ as small as necessary, such that on the complement $U \backslash Z_{0}$ the leading coefficient admits a lower estimate given by Lemma 1 , in terms of $\mathbf{b}_{K, U}\left(a_{0}\right) \leqslant \ln _{+}\|L\|$. Then Theorem 1 can be applied to the unitary operator $\tilde{L}=a_{0}^{-1}(t) L$ in the same way as in [2], since one may control effectively the magnitude of coefficients of $\tilde{L}$ on $U \backslash Z_{0}$.

\subsection{Oscillation in polynomial envelopes: demonstration of Theorems 2 and $2^{\prime}$}

We use the following result proved in [3]. Let $L$ be an irreducible operator (in the sense described in $\S 1.5$ ), let $K^{\prime} \Subset U^{\prime} \Subset U$ be two nested CP-gons and let $f_{1}, \ldots, f_{n}$ be any fundamental system of solutions of the equation $L u=0$.

Consider the monomials $F_{j k l}(t)=t^{l} f_{j}^{(k-1)}(t)$ for $j, k=1, \ldots, n, l=0,1, \ldots, d$ ordered, say, lexicographically, and let $W_{s}(t)$ be the Wronskian of the first $s$ monomials, $s=1, \ldots, n^{2}(d+1)$. The monomials $F_{j k l}$ span the polynomial $d$-envelope of $L$.

LeMma 6 (see [3]). If $L$ is an irreducible operator, then the Bernstein indices of the Wronskians $W_{s}$ for all $s=1, \ldots, n^{2}(d+1)$ allow the following upper estimate, which grows at most exponentially with $d$ :

$$
\forall s=1, \ldots, n^{2}(d+1) \quad \mathbf{5}_{K^{\prime}, U^{\prime}}\left(W_{s}\right) \leqslant \exp \left(c_{1} d\right), \quad c_{1}=c_{1}\left(K^{\prime}, U^{\prime}\right) .
$$

The differential operator $\mathscr{L}_{d}$ annulating all monomials, $F_{j k l}$ for $l=1, \ldots, d$, and hence all their linear combinations, can be written in the Frobenius form

$$
\begin{aligned}
\mathscr{L}_{d} & =\frac{W_{N}}{W_{N-1}} \cdot \partial \cdot \frac{W_{n-1}^{2}}{W_{N} W_{N-2}} \cdot \partial \cdot \frac{W_{N-2}^{2}}{W_{N-1} W_{N-3}} \cdot \partial \cdots \partial \cdot \frac{W_{1}^{2}}{W_{2} W_{0}} \cdot \partial \cdot \frac{W_{0}}{W_{1}} \\
& =R_{N} \circ \partial \circ R_{N-1} \circ \partial \circ \cdots \circ R_{1} \circ \partial \circ R_{0},
\end{aligned}
$$

where $N=N(d)=n^{2}(d+1)$ is the total number of monomials; the order of this operator is $N=O(d)$. 
Now the complex Rolle theorem in the general form can be applied. The operator $\mathscr{L}_{d}$ is decomposed as a product of $2 N+1$ operators of orders alternating between 0 and 1. Choose a sequence of $2 N+1 \mathrm{CP}$-gons $K \Subset \cdots \Subset K_{i+1} \Subset K_{i} \Subset \cdots \Subset K^{\dagger} \Subset U^{\prime} \Subset U$ with boundaries $\Gamma_{i}$ : this can be done with $\operatorname{gap}\left(K_{i+1}, K_{i}\right)=O(1 / N)$. If $f$ is analytic in $U^{\prime}$, then each partial result

$$
f_{s}=R_{s} \circ \partial \circ R_{s-1} \circ \cdots \circ R_{0} f
$$

can be represented as a meromorphic function,

$$
f_{s}=\frac{h_{s}}{g_{s}}, \quad g_{s}=W_{0}^{2^{s}} W_{1}^{2^{s-1}} \cdots W_{s},
$$

hence by the complex Rolle theorem $4^{\prime}$ the operators of differentiation cannot incur the loss of more than $\exp O(d)+$ const $\cdot \ln N=\exp O(d)$ units of the Bernstein index of the numerators. The impact of applying $R_{s}$ is well described by Lemmas 3 and 4 . Finally we conclude that the operator $\mathscr{L}_{d}$ cannot cancel more than $d \exp O(d)=$ $\exp O(d)$ units of the Bernstein index, which proves Theorem 2 in the irreducible case, since any function from the polynomial $d$-envelope is annulated by $\mathscr{L}_{d}$, and $\mathrm{b}_{K, U}(0)=0$.

As for the general case of the composition of an irreducible operator and several operators of order 1 , it is shown in [6] that for any function $f$ from the polynomial $d$-envelope of $L P_{k} \cdots P_{2} P_{1}$ one can explicitly construct a differential operator of the form

$$
Q_{s} \circ \partial \circ Q_{s-1} \circ \partial \circ \cdots \circ Q_{1} \circ \partial \circ Q_{0},
$$

in general depending on $f$, with rational factors $Q_{j}$ of degree at most $O(d)$, that would take $f$ into a function from the polynomial envelope of $L^{\prime}=L P_{k} \cdots P_{2}$. This immediately implies an upper estimate for the Bernstein index.

\subsection{Zeros of derivatives: proof of Theorem 3}

As already mentioned in $\S 1$, for any function satisfying an equation $L f=0$ with $L=\Sigma_{0}^{n} a_{j} \partial^{n-j} \in \mathbb{C}(t)[\partial]$ with ord $L=n$, its $d$ th derivative can be expressed as a linear combination of $f, f^{\prime}, \ldots, f^{(n-1)}$ with rational coefficients from $\mathbb{C}(t)$. In this section we show that for a fixed $L$ the degrees of the coefficients will be at most $O(d)$. This will reduce Theorem 3 to Theorem 2 .

If one wishes to differentiate the identity

$$
f^{(n)}=\sum_{j=0}^{n-1} r_{j}(t) f^{(j)} \quad \text { for } r_{j}=-\frac{a_{n-j}}{a_{0}},
$$

then, since each differentiation in general doubles the degree of a rational function, $\operatorname{deg} \phi^{\prime} \leqslant 2 \operatorname{deg} \phi$ for $\phi \in \mathbb{C}(t)$, the straightforward estimate for the growth of degrees of coefficients will be exponential in $d$. In order to show that this growth is at most linear, we take into account the fact that the singularities of all the derivatives will remain the same. We prove by induction that

$$
\begin{gathered}
a_{0}(t)^{d-n+1} f^{(d)}=\sum_{j=0}^{n-1} p_{j, d}(t) f^{(j)}(t) \quad \forall d=n, n+1, \ldots, \\
a_{0}, p_{j, d} \in \mathbb{C}[t], \quad \operatorname{deg} p_{j, d} \leqslant(d-n+1) \max \operatorname{deg} a_{j}=O(d) .
\end{gathered}
$$


Indeed, for $d=n$ this is the differential equation $L f=0$. Differentiating this identity for an arbitrary $d$ and multiplying it by $a_{0}$, we obtain

$$
\begin{aligned}
(d-n+1) a_{0}^{d-n+1} a_{0}^{\prime} f^{(d)}+a_{0}^{d-n+2} f^{(d+1)} & =\sum_{j=0}^{n-1} a_{0}\left(p_{j, d}^{\prime}+p_{j-1, d}\right) f^{(j)}+a_{0} p_{n-1, d} f^{(n)} \\
& =\sum_{j=0}^{n-1} a_{0}\left(p_{j, d}^{\prime}+p_{j-1, d}\right) f^{(j)}+\sum_{j=0}^{n-1} p_{n-1, d} p_{j, n} f^{(j)},
\end{aligned}
$$

and hence

$$
a_{0}^{d-n+2} f^{(d+1)}=\sum_{j=0}^{n-1}\left(a_{0}\left(p_{j, d}^{\prime}+p_{j-1, d}\right)+p_{n-1, d} p_{j, n}-(d-n+1) a_{0}^{\prime} p_{j, d}\right) f^{(j)} .
$$

Thus the degrees of the coefficients $p_{j, d+1}$ compared to those of $p_{j, d}$ will be increased by at most $\max \left(\operatorname{deg} a_{0}, \operatorname{deg} p_{j, n}\right)$; the latter number is the maximum of the degrees of the coefficients of $L$ put into the unitary form.

Thus we see that, after multiplication by an appropriate rational function $a_{0}^{d-n+1}$ which does not change the number of zeros, the derivative $f^{(d)}$ belongs to the polynomial $O(d)$-envelope of the operator $L$. Theorem 2 now gives an upper estimate for the number of isolated zeros of $f^{(d)}$.

\section{References}

1. S. Bernstein, 'Leçons sur les propriétés extrémales et la meilleure approximation des fonctions analytiques d'une variable réelle', Paris, 1926; reprinted in S. Bernstein and C. DE LA VAlLÉE Poussin, l'Approximation (Chelsea, Bronx, 1970).

2. YU. IL'yashenKo and S. YAKOVENKo, 'Counting the number of zeros of analytic functions satisfying linear ordinary differential equations', J. Differential Equations 126 (1996) 87-105.

3. Yu. IL'yashenko and S. Yakovenko, 'Double exponential estimate for the number of zeros of complete Abelian integrals and rational envelopes of linear differential equations with an irreducible monodromy group', Invent. Math. 121 (1995) 613-650.

4. A. KhovanskiĬ, Fewnomials, American Mathematical Society Colloquium Publications (American Mathematical Society, Providence, 1991).

5. D. Novikov and S. Yakovenko, 'Une borne simplement exponentielle pour le nombre de zéros réels isolés des intégrales complètes abéliennes', C.R. Acad. Sci. Paris Sér. I 320 (1995) 853-858.

6. D. Novikov and S. Yakovenko, 'Simple exponential estimate for the number of zeros of complete Abelian integrals', Ann. Inst. Fourier (Grenoble) 45 (1995) 897-927.

7. G. Petrov, 'On the number of zeros of complete Abelian integrals', Functional Anal. Appl. 18 (1984) 73-74; 'Complex zeros of an elliptic integral', ibid. 20 (1986) 46-49; 'Nonoscillation of elliptic integrals', ibid. 24 (1990) 45-50.

8. M. VoORHOEVE, 'On the oscillation of exponential polynomials', Math. Z. 151 (1976) 277-294.

9. M. VoORhoeve, 'A generalization of Descartes' rule', J. London Math. Soc. (2) 20 (1979) 446-456.

10. S. Yakovenko, 'Abelian integrals as rational envelopes', Nonlinearity 7 (1994) 1237-1250.

Department of Theoretical Mathematics

The Weizmann Institute of Science

Rehovot 76100

Israel

E-mail address:dmitri@math.weizmann.ac.il yakov@math.weizmann.ac.il 